\title{
Coupled cluster calculations of ground and excited states of nuclei
}

\author{
K. Kowalski ${ }^{1}$, D.J. Dean ${ }^{2}$, M. Hjorth-Jensen ${ }^{3}$, T. Papenbrock ${ }^{2,4}$, and P. Piecuch ${ }^{1,5}$ \\ ${ }^{1}$ Department of Chemistry, Michigan State University, East Lansing, MI 48824 \\ ${ }^{2}$ Physics Division, Oak Ridge National Laboratory, P.O. Box 2008, Oak Ridge, TN 37831 \\ ${ }^{3}$ Department of Physics and Center of Mathematics for Applications, University of Oslo, N-0316 Oslo, Norway \\ ${ }^{4}$ Department of Physics and Astronomy, University of Tennessee, Knoxville, TN 37996 USA and \\ ${ }^{5}$ Department of Physics and Astronomy, Michigan State University, East Lansing, MI 48824
}

(Dated: February 9, 2020)

\begin{abstract}
The standard and renormalized coupled cluster methods with singles, doubles, and noniterative triples and their generalizations to excited states, based on the equation of motion coupled cluster approach, are applied to the ${ }^{4} \mathrm{He}$ and ${ }^{16} \mathrm{O}$ nuclei. A comparison of coupled cluster results with the results of the exact diagonalization of the Hamiltonian in the same model space shows that the quantum chemistry inspired coupled cluster approximations provide an excellent description of ground and excited states of nuclei. The bulk of the correlation effects is obtained at the coupled cluster singles and doubles level. Triples, treated noniteratively, provide the virtually exact description.
\end{abstract}

The description of finite nuclei requires an understanding of both ground- and excited-state properties based on a given nuclear Hamiltonian. While much progress has been made in employing the Green's Function Monte Carlo 1] and no-core shell model 2] techniques, these methods have apparent limitations to light nuclei. Given that present nuclear structure research facilities and the proposed Rare Isotope Accelerator will continue to open significant territory into regions of medium-mass and heavier nuclei, it becomes imperative to investigate methods that will allow for a description of medium-mass systems. Coupled cluster theory is a particularly promising candidate for such an endeavor due to its enormous success in quantum chemistry [3, 4, 5, 6, 7, 8, 9].

Coupled cluster theory originated in nuclear physics 10, 11] around 1960. Early studies in the seventies [12] probed ground-state properties in limited spaces with free nucleon-nucleon interactions available at the time. The subject was revisited only recently by Guardiola et al. 13], for further theoretical development, and by Mihaila and Heisenberg 14, for coupled cluster calculations using realistic two- and three-nucleon bare interactions and expansions in the inverse particle-hole energy spacings. However, much of the impressive development in coupled cluster theory made in quantum chemistry in the last 15-20 years [5, 6, 7, 8, 9] still awaits applications to the nuclear many-body problem.

In this Letter, we apply quantum chemistry inspired coupled cluster methods [3, 4, 5, 6, 7, 8, 9, 15, 16] to finite nuclei. We show that the coupled cluster approach is numerically inexpensive and accurate by comparing our results for ${ }^{4} \mathrm{He}$ with results from exact diagonalization in a model space consisting of four major oscillator shells. For the first time, we apply coupled cluster theory to excited states in nuclei, exploiting the equation of motion coupled cluster formalism [15, 16]. We discuss several approximations within coupled cluster theory and also compute the ground state of the ${ }^{16} \mathrm{O}$ nucleus within the same model space. We remind the reader that certain acronyms have become standard in quantum chemistry. For this reason, we use the same abbreviations in this Letter.

Coupled cluster theory is based on an exponential ansatz for the ground-state wave function $\left|\Psi_{0}\right\rangle=$ $\exp (T)|\Phi\rangle$. Here $T$ is the cluster operator and $|\Phi\rangle$ is the reference determinant. In the CCSD ("coupled cluster with singles and doubles") method, we truncate the many-body expansion of the cluster operator $T$ at two-body components. The truncated cluster operator $T^{(\mathrm{CCSD})}$, used in the CCSD calculations, has the form 17]: $T^{(\mathrm{CCSD})}=T_{1}+T_{2}$. Here $T_{1}=$ $\sum_{i, a} t_{a}^{i} a^{a} a_{i}$ and $T_{2}=\frac{1}{4} \sum_{i j, a b} t_{a b}^{i j} a^{a} a^{b} a_{j} a_{i}$ are the singly and doubly excited clusters, with indices $i, j, k(a, b, c)$ designating the single-particle states occupied (unoccupied) in the reference Slater determinant $|\Phi\rangle$ and $a^{p}\left(a_{p}\right)$ representing the creation (annihilation) operators. We determine the singly and doubly excited cluster amplitudes $t_{a}^{i}$ and $t_{a b}^{i j}$, defining $T_{1}$ and $T_{2}$, respectively, by solving the nonlinear system of algebraic equations, $\left\langle\Phi_{i}^{a}\left|\bar{H}^{(\mathrm{CCSD})}\right| \Phi\right\rangle=0,\left\langle\Phi_{i j}^{a b}\left|\bar{H}^{(\mathrm{CCSD})}\right| \Phi\right\rangle=0$, where $\bar{H}^{(\mathrm{CCSD})}=\exp \left(-T^{(\mathrm{CCSD})}\right) H \exp \left(T^{(\mathrm{CCSD})}\right)$ is the similarity-transformed Hamiltonian and $\left|\Phi_{i}^{a}\right\rangle$ and $\left|\Phi_{i j}^{a b}\right\rangle$ are the singly and doubly excited Slater determinants, respectively. Once $T_{1}$ and $T_{2}$ amplitudes are determined, we calculate the ground-state CCSD energy $E_{0}^{(\mathrm{CCSD})}$ as $\left\langle\Phi\left|\bar{H}^{(\mathrm{CCSD})}\right| \Phi\right\rangle$. For the excited states $\left|\Psi_{K}\right\rangle$ and energies $E_{K}^{(\mathrm{CCSD})}(K>0)$, we apply the EOMCCSD ("equation of motion CCSD") approximation, in which

$$
\left|\Psi_{K}\right\rangle=R_{K}^{(\mathrm{CCSD})} \exp \left(T^{(\mathrm{CCSD})}\right)|\Phi\rangle .
$$

Here $R_{K}^{(\mathrm{CCSD})}=R_{0}+R_{1}+R_{2}$ is a sum of the reference $\left(R_{0}\right)$, one-body $\left(R_{1}\right)$, and two-body $\left(R_{2}\right)$ components that are obtained by diagonalizing the similaritytransformed Hamiltonian $\bar{H}^{\text {(CCSD) }}$ in the same space of singly and doubly excited determinants $\left|\Phi_{i}^{a}\right\rangle$ and $\left|\Phi_{i j}^{a b}\right\rangle$ as used in the ground-state CCSD calculations [15, 16].

The CCSD and EOMCCSD methods are expected to 
describe the bulk of the correlation effects with inexpensive computational steps that scale as $n_{o}^{2} n_{u}^{4}$, where $n_{o}\left(n_{u}\right)$ is the number of occupied (unoccupied) singleparticle orbitals. While the inclusion of triply excited clusters $T_{3}$ and three-body excitation operators $R_{3}$ increases the accuracy of the method, the resulting full CCSDT ("T" stands for "triples") 18 and EOMCCSDT [19] methods scale as $n_{o}^{3} n_{u}^{5}$ and are rather expensive. For this reason, we add the a posteriori corrections due to triples to the CCSD/EOMCCSD energies, which require $n_{o}^{3} n_{u}^{4}$ noniterative steps. The ground- and excited-state triples corrections, $\delta_{0}$ and $\delta_{K}(K>0)$, respectively, are calcultated with the CR-CCSD $(\mathrm{T})$ ("completely renormalized $\operatorname{CCSD}(\mathrm{T}) ")$ approach [8, 9, 20, 21] in which

$$
\delta_{K}=\frac{1}{36} \sum_{i j k, a b c}\left\langle\tilde{\Psi}_{K} \mid \Phi_{i j k}^{a b c}\right\rangle \mathcal{M}_{a b c}^{i j k}(K) / \Delta_{K}(K \geq 0) .
$$

Here $\left|\Phi_{i j k}^{a b c}\right\rangle$ are the triply excited determinants and $\mathcal{M}_{a b c}^{i j k}(K)$ are the generalized moments of the CCSD $(K=0)$ and EOMCCSD $(K>0)$ equations 20, 21, 22],

$$
\mathcal{M}_{a b c}^{i j k}(K)=\left\langle\Phi_{i j k}^{a b c}\left|\bar{H}^{(\mathrm{CCSD})} S_{K}^{(\mathrm{CCSD})}\right| \Phi\right\rangle,
$$

where $S_{0}^{(\mathrm{CCSD})}=1$ and $S_{K}^{(\mathrm{CCSD})}=R_{K}^{(\mathrm{CCSD})}$ for $K>0$. They can be calculated using the CCSD and EOMCCSD cluster and excitation operators $T^{(\mathrm{CCSD})}$ and $R_{K}^{(\mathrm{CCSD})}$, respectively. The $\Delta_{K}$ denominators are defined as

$$
\Delta_{K}=\left\langle\tilde{\Psi}_{K}\left|S_{K}^{(\mathrm{CCSD})} \exp \left(T^{(\mathrm{CCSD})}\right)\right| \Phi\right\rangle .
$$

The states $\left|\tilde{\Psi}_{K}\right\rangle$ in Eqs. (2) and (4) include the leading triples contributions resulting from the perturbative analysis of the CCSDT and EOMCCSDT equations. We have $\left|\tilde{\Psi}_{0}\right\rangle=\bar{P} \exp \left(T^{(\mathrm{CCSD})}+\tilde{T}_{3}\right)|\Phi\rangle$ and $\left|\tilde{\Psi}_{K}\right\rangle=\bar{P}\left(R_{K}^{(\mathrm{CCSD})}+\tilde{R}_{3}\right) \exp \left(T^{(\mathrm{CCSD})}\right)|\Phi\rangle$ for $K>0$, where $\bar{P}$ is a projection operator on the subspace spanned by the reference $|\Phi\rangle$ and singly, doubly, and triply excited determinants. The most complete forms of $\tilde{T}_{3}$ and $\tilde{R}_{3}$ defining the CR-CCSD $(\mathrm{T}), \mathrm{c}$ approximation are 8,21$]$

$$
\begin{aligned}
& \tilde{T}_{3}=\frac{1}{36} \sum_{i j k, a b c}\left(\mathcal{M}_{a b c}^{i j k}(0) / D_{i j k}^{a b c}(0)\right) a^{a} a^{b} a^{c} a_{k} a_{j} a_{i}, \\
& \tilde{R}_{3}=\frac{1}{36} \sum_{i j k, a b c}\left(\mathcal{M}_{a b c}^{i j k}(K) / D_{i j k}^{a b c}(K)\right) a^{a} a^{b} a^{c} a_{k} a_{j} a_{i},(6)
\end{aligned}
$$

where $D_{i j k}^{a b c}(K)=E_{K}^{(\mathrm{CCSD})}-\left\langle\Phi_{i j k}^{a b c}\left|\bar{H}^{(\mathrm{CCSD})}\right| \Phi_{i j k}^{a b c}\right\rangle$. In the case of the ground-state calculations, we also consider simplified variants of the CR-CCSD(T) theory, termed CR-CCSD(T), a and CR-CCSD(T), b. In the case of CR$\operatorname{CCSD}(\mathrm{T}), \mathrm{b}$, the perturbative denominator $D_{i j k}^{a b c}(0)$ is replaced by $-\left\langle\Phi_{i j k}^{a b c}\left|\bar{H}_{1}^{(\mathrm{CCSD})}\right| \Phi_{i j k}^{a b c}\right\rangle$, where $\bar{H}_{1}^{(\mathrm{CCSD})}$ is the one-body part of $\bar{H}^{\text {(CCSD) }}$. For CR-CCSD(T), a we replace $D_{i j k}^{a b c}(0)$ by the standard many-body perturbation theory (MBPT) triples denominator $\left(\epsilon_{i}+\epsilon_{j}+\epsilon_{k}-\epsilon_{a}-\right.$ $\epsilon_{b}-\epsilon_{c}$ ), where $\epsilon_{i}$ and $\epsilon_{a}$ are the diagonal elements of the Fock matrix. Very accurate results for the excitation energies $E_{K}-E_{0}$ of many-electron systems are obtained if we use the complete CR-CCSD $(\mathrm{T}), \mathrm{c}$ theory to calculate the energies of excited states and the $\mathrm{CR}-\operatorname{CCSD}(\mathrm{T}), \mathrm{b}$ approximation for the ground-state energy 21]. For the ground states, it may sometimes be worthwhile to replace the $\Delta_{0}$ denominator, Eq. (4), which renormalizes the triples correction $\delta_{0}$ by 1 , since $\Delta_{0}$ equals 1 plus terms of the second MBPT order or higher 20]. We indicate this by using acronyms, such as CR-CCSD $(\mathrm{T}), \mathrm{c} / \Delta_{0}=1$ (as opposed to CR-CCSD(T),c, where $\Delta_{0}$ is included).

We use the Idaho-A nucleon-nucleon potential 23. which was produced using techniques of chiral effective field theory 24, 25]. Modern two-nucleon interactions, such as Idaho-A, include short-range repulsive cores that require calculations in extremely large model spaces to reach converged results 14]. In order to remove the hard-core part of the interaction from the problem and thereby allow for realistic calculations in manageable model spaces, we renormalize the interactions through a $G$-matrix procedure for use in the $0 s-0 p-0 d 1 s$ $0 f 1 p$ oscillator basis. Our Hamiltonian is thus given by $H=t+G(\tilde{\omega})$, where $\tilde{\omega}$ is the $G$-matrix starting energy. We use a simple procedure described in Ref. [26] to alleviate the starting-energy dependence of the $G$-matrix in orbitals below the Fermi surface. We also modify the Hamiltonian by adding to it the center-of-mass Hamiltonian times a Lagrange multiplier $\beta_{\text {c.m. }}$. Thus, our Hamiltonian becomes $H^{\prime}=H+\beta_{\text {c.m. }} H_{\text {c.m. }}$. We choose $\beta_{\text {c.m. }}$. such that the expectation value of $H_{\text {c.m. }}=0.0 \mathrm{MeV}$. Details may be found in Ref. 27].

We tested the performance of the above coupled cluster approximations in the context of the nuclear many-body problem by applying them to two closed-shell nuclei, ${ }^{4} \mathrm{He}$ and ${ }^{16} \mathrm{O}$, in the one-particle space of four major oscillator shells. Shell model diagonalization provided an exact answer for a given Hamiltonian in the ${ }^{4} \mathrm{He}$ case. Comparing the exact ground- and excited-state energies resulting from the diagonalization of the Hamiltonian in the small model space with the coupled cluster energies obtained in the same model space, we can assess the usefulness of various coupled cluster approximations in calculations for atomic nuclei. In particular, we can learn about the possible role of triply excited clusters in an accurate description of ground and excited states without confusing the inaccuracies resulting from the inadequate treatment of the many-body problem by a given coupled cluster approximation with other sources of error.

We report our results for the ground-state energy of ${ }^{4} \mathrm{He}$ in Table【 We used two types of reference determinants $|\Phi\rangle$ : one constructed from the lowest-energy oscillator states and the Hartree-Fock determinant. Throughout the table we see that the results obtained in the oscillator basis are lower in energy when compared to those obtained in the Hartree-Fock basis. The two best meth- 
ods in the oscillator basis are $\operatorname{CR}-\operatorname{CCSD}(\mathrm{T}), \mathrm{a} / \Delta_{0}=1$ and $\operatorname{CR}-\operatorname{CCSD}(\mathrm{T}), \mathrm{c} / \Delta_{0}=1$. They yield results within $40 \mathrm{keV}$ and $300 \mathrm{keV}$ of the full configuration interaction (CI) diagonalization problem, respectively. The CR$\operatorname{CCSD}(\mathrm{T}), \mathrm{a} / \Delta_{0}=1$ approach applied to the oscillator basis overshoots the exact result, which is a consequence of using the standard MBPT denominators in the definition of $\tilde{T}_{3}$, Eq. (5). The CR-CCSD(T), a approach, in which the triples correction $\delta_{0}$ is renormalized via the presence of the $\Delta_{0}$ denominator in Eq. (2), is more stable in this regard, providing the upper bound to the energy, although the $600 \mathrm{keV}$ error obtained with the CR$\operatorname{CCSD}(\mathrm{T})$, a method is not as impressive as the $40 \mathrm{keV}$ error obtained with $\operatorname{CR}-\operatorname{CCSD}(\mathrm{T}), \mathrm{a} / \Delta_{0}=1$. In general, the CR-CCSD $(\mathrm{T})$ results are considerably more accurate than the results of the CCSD calculations, in which $T_{3}$ is ignored, although the CCSD approach describes the bulk of the correlation effects, reducing the large $16.273 \mathrm{MeV}$ error obtained by calculating $\langle\Phi|H| \Phi\rangle$ with the oscillator reference $|\Phi\rangle$ to $1.5 \mathrm{MeV}$. The effectiveness of the CCSD approach can also be illustrated by comparing the CCSD energy with the results of truncated shell-model calculations (CISD), in which the Hamiltonian is diagonalized in the same space of singly and doubly excited determinants as used in the CCSD caculations. The costs of the CISD and CCSD calculations are almost identical (both are $n_{o}^{2} n_{u}^{4}$ procedures), and yet the error in the CISD energy obtained in the oscillator basis is twice as large as the error obtained with CCSD. The noniterative triples corrections defining the CR-CCSD(T) approaches reduce these errors to as little as $40 \mathrm{keV}$, which is a lot better than the $1.3 \mathrm{MeV}$ error in the CISDT calculations, where the Hamiltonian is diagonalized in the much larger space of all singly, doubly, and triply excited determinants. This demonstrates the advantages of coupled cluster methods over the diagonalization techniques. Similar observations apply to the Hartree-Fock basis, although the coupled cluster results obtained with this basis are not as good as those obtained with the oscillator basis. For example, the best result in the Hartree-Fock basis, obtained with CR-CCSD $(\mathrm{T}), \mathrm{c} / \Delta_{0}=1$, is $700 \mathrm{keV}$ above the exact result. This suggests that we may be better off by using the oscillator basis in coupled cluster calculations. On the other hand, the $\mathrm{CR}-\mathrm{CCSD}(\mathrm{T})$ results obtained in the Hartree-Fock basis are not unreasonable, allowing us to contemplate the use of the Hartree-Fock basis in coupled cluster calculations for open-shell nuclei. (This would parallel the Hartree-Fock-based coupled cluster calculations for open-shell electronic states in chemistry.)

We used the EOMCCSD method and its CR-CCSD(T) extension to compute excited states. To our knowledge, this is the first time that nuclear excited states are computed using coupled cluster methods. The results for ${ }^{4} \mathrm{He}$ are given in Table III The low-lying $J=1$ state most likely results from the center-of-mass contamination which we have removed only from the ground state. The
TABLE I: The ground-state energies of ${ }^{4} \mathrm{He}$ calculated using the oscillator (Osc) and Hartree-Fock (HF) basis states. Units are $\mathrm{MeV}$. The reference energies $\left\langle\Phi\left|H^{\prime}\right| \Phi\right\rangle$ are -7.211 (Osc) and $-10.520(\mathrm{HF}) \mathrm{MeV}$.

\begin{tabular}{|crc|}
\hline Method & Osc & HF \\
\hline CCSD & -21.978 & -21.385 \\
CR-CCSD $(\mathrm{T}), \mathrm{a}$ & -22.841 & -22.395 \\
CR-CCSD $(\mathrm{T}), \mathrm{a} / \Delta_{0}=1$ & -23.524 & -22.711 \\
CR-CCSD $(\mathrm{T}), \mathrm{b}$ & -22.396 & -22.179 \\
$\mathrm{CR}-\mathrm{CCSD}(\mathrm{T}), \mathrm{b} / \Delta_{0}=1$ & -22.730 & -22.428 \\
$\mathrm{CR}-\mathrm{CCSD}(\mathrm{T}), \mathrm{c}$ & -22.630 & -22.450 \\
$\mathrm{CR}-\mathrm{CCSD}(\mathrm{T}), \mathrm{c} / \Delta_{0}=1$ & -23.149 & -22.783 \\
$\mathrm{CISD}$ & -20.175 & -20.801 \\
CISDT & -22.235 & - \\
Exact & -23.484 & -23.484 \\
\hline
\end{tabular}

TABLE II: The excitation energies of ${ }^{4} \mathrm{He}$ calculated using the oscillator basis states (in $\mathrm{MeV}$ ).

\begin{tabular}{|lcccc|}
\hline State & EOMCCSD & CR-CCSD $(\mathrm{T})^{a}$ & CISD & Exact \\
\hline $\mathrm{J}=1$ & 11.791 & 12.044 & 17.515 & 11.465 \\
$\mathrm{~J}=0$ & 21.203 & 21.489 & 24.969 & 21.569 \\
$\mathrm{~J}=2$ & 22.435 & 22.650 & 24.966 & 22.697 \\
\hline
\end{tabular}

${ }^{a}$ The difference of the CR-CCSD(T),c energy of excited state and the CR-CCSD(T),b energy of the ground state.

$J=0$ and $J=2$ states calculated using EOMCCSD and CR-CCSD $(\mathrm{T})$ are in excellent agreement with the exact results. For these two states, the EOMCCSD approach provides the relatively small, $0.3-0.4 \mathrm{MeV}$, errors, which are further reduced by the CR-CCSD $(\mathrm{T})$ triples corrections to $<0.1 \mathrm{MeV}$. Based on the experience with the equation of motion coupled cluster methods in chemistry 5, 8, 15, 19, 21, 22], the very good performance of the EOMCCSD approach for the lowest-energy excited states of ${ }^{4} \mathrm{He}$ can be understood if we realize that these states are dominated by single-particle excitations. Again, a comparison of the EOMCCSD and CISD results shows that coupled cluster theory offers much higher accuracies compared to truncated diagonalization of similar numerical effort. According to the experiment, the lowest lying $0^{+}$state in ${ }^{4} \mathrm{He}$ is a resonance at an excitation of $20.21 \mathrm{MeV}$ and a width of $0.5 \mathrm{MeV}$, while the first $J=2$ state lies at $21.84 \mathrm{MeV}$ and has a width of $2 \mathrm{MeV}$. We have not identified the parity of our calculated states, but it seems to us that we will be able to model the excitations in ${ }^{4} \mathrm{He}$ and other nuclei using coupled cluster theory. Our results in Table II are indicative of the accuracies we may expect from such calculations.

We also applied the CCSD and CR-CCSD(T) methods to ${ }^{16} \mathrm{O}$. Table III shows the total ground-state energy 
TABLE III: The ground-state energy of ${ }^{16} \mathrm{O}$ calculated using various coupled cluster methods and oscillator basis states. The reference energy $\left\langle\Phi\left|H^{\prime}\right| \Phi\right\rangle$ is $-109.452 \mathrm{MeV}$.

\begin{tabular}{|cr|}
\hline Method & Energy \\
\hline CCSD & -139.310 \\
CR-CCSD $(\mathrm{T}), \mathrm{a}$ & -139.465 \\
$\mathrm{CR}-\mathrm{CCSD}(\mathrm{T}), \mathrm{a} / \Delta_{0}=1$ & -139.621 \\
$\mathrm{CR}-\mathrm{CCSD}(\mathrm{T}), \mathrm{b}$ & -139.375 \\
$\mathrm{CR}-\mathrm{CCSD}(\mathrm{T}), \mathrm{b} / \Delta_{0}=1$ & -139.440 \\
$\mathrm{CR}-\mathrm{CCSD}(\mathrm{T}), \mathrm{c}$ & -139.391 \\
$\mathrm{CR}-\mathrm{CCSD}(\mathrm{T}), \mathrm{c} / \Delta_{0}=1$ & -139.467 \\
$\mathrm{CISD}$ & -131.887 \\
$\mathrm{CISDT}$ & -135.489 \\
$\mathrm{CISDTQ}$ & -138.387 \\
\hline
\end{tabular}

values obtained with the CCSD and CR-CCSD(T) approaches. As in the ${ }^{4} \mathrm{He}$ case, coupled cluster methods recover the bulk of the correlation effects, producing the results of the CISDTQ, or better, quality. CISDTQ stands for the expensive shell-model diagonalization in a huge space spanned by the reference and all singly, doubly, triply, and quadruply excited determinants (the most expensive steps of CISDTQ scale as $\left.n_{o}^{4} n_{u}^{6}\right)$. To understand this result, we note that the CCSD $T_{1}$ and $T_{2}$ amplitudes are similar in order of magnitude. (For an oscillator basis, both $T_{1}$ and $T_{2}$ contribute to the first-order MBPT wave function.) Thus, the $T_{1} T_{2}$ disconnected triples are large, much larger than the $T_{3}$ connected triples, and the difference between the CISDT (CI singles, doubles, and triples) and CISD energies is mostly due to $T_{1} T_{2}$. The small $T_{3}$ effects, as estimated by CR-CCSD $(\mathrm{T})$, are consistent with the CI diagonalization calculations. If the $T_{3}$ corrections were large, we would observe a significant lowering of the CCSD energy, far below the CISDTQ result. The CISDTQ diagonalization is not size-extensive, while the CCSD and CR-CCSD $(\mathrm{T}) / \Delta_{0}=1$ methods maintain this property. Moreover, the CCSD and CR-CCSD $(\mathrm{T})$ methods bring the nonnegligible higher-than-quadruple excitations, such as $T_{1}^{3} T_{2}, T_{1} T_{2}^{2}$, and $T_{2}^{3}$, which are not present in CISDTQ. It is, therefore, quite likely that the CR-CCSD $(\mathrm{T})$ results are very close to the results of the exact diagonalization, which cannot be performed.

In summary, we used the quantum chemistry inspired coupled cluster approximations to calculate the ground and excited states of the ${ }^{4} \mathrm{He}$ and ${ }^{16} \mathrm{O}$ nuclei. By comparing coupled cluster results with the exact results obtained by diagonalizing the Hamiltonian in the same model space, we demonstrated that relatively inexpensive coupled cluster approximations recover the bulk of the nucleon correlation effects in ground- and excitedstate nuclei. These results are a strong motivation to further develop coupled cluster methods for the nuclear many-body problem, so that accurate $a b$ initio calculations for small- and medium-size nuclei become as routine as the molecular electronic structure calculations.

Supported by the U.S. Department of Energy under Contract Nos. DE-FG02-96ER40963 (University of Tennessee), DE-AC05-00OR22725 with UT-Battelle, LLC (Oak Ridge National Laboratory), DE-FG02-01ER15228 (Michigan State University), the Research Council of Norway, and the Alfred P. Sloan Foundation.

[1] S. C. Pieper, K. Varga, and R. B. Wiringa, Phys. Rev. C 66, 44310 (2003).

[2] P. Navratil, J. P. Vary, and B. R. Barrett, Phys. Rev. Lett. 84, 5728 (2000).

[3] J. Č́źžek, J. Chem. Phys. 45, 4256 (1966).

[4] J. Č́žzek, Adv. Chem. Phys. 14, 35 (1969).

[5] R. J. Bartlett, in Modern Electronic Structure Theory, edited by D. R. Yarkony (World Scientific, Singapore, 1995), vol. 1, pp. 1047-1131.

[6] J. Paldus and X. Li, Adv. Chem. Phys. 110, 1 (1999).

[7] T. D. Crawford and H. F. Schaefer III, Rev. Comp. Chem. 14, 33 (2000).

[8] P. Piecuch, K. Kowalski, I.S.O. Pimienta, and M.J. McGuire, Int. Rev. Phys. Chem. 21, 527 (2002).

[9] P. Piecuch, K. Kowalski, P.-D. Fan, and I.S.O. Pimienta, in Topics in Theoretical Chemical Physics, edited by J. Maruani, R. Lefebvre, and E. Brändas (Kluwer, Dordrecht, 2003), vol. 12 of Progress in Theoretical Chemistry and Physics, pp. 119-206.

[10] F. Coester, Nucl. Phys. 7, 421 (1958).

[11] F. Coester and H. Kümmel, Nucl. Phys. 17, 477 (1960).

[12] H. Kümmel, K. H. Luhrmann, and J. G. Zabolitzky, Phys. Rep. 36, 1 (1978), and references therein.

[13] R. Guardiola et al., Nucl. Phys. A 609, 218 (1996).

[14] J. H. Heisenberg and B. Mihaila, Phys. Rev. C 59, 1440 (1999); B. Mihaila and J. H. Heisenberg, Phys. Rev. C 61, 054309 (2000).

[15] J. F. Stanton and R. J. Bartlett, J. Chem. Phys. 98, 7029 (1993).

[16] P. Piecuch and R. J. Bartlett, Adv. Quantum Chem. 34, 295 (1999).

[17] G. D. Purvis and R. J. Bartlett, J. Chem. Phys. 76, 1910 (1982).

[18] J. Noga and R. J. Bartlett, J. Chem. Phys. 86, 7041 (1987); 89, 3401 (1988) (erratum); G.E. Scuseria and H.F. Schaefer III, Chem. Phys. Lett. 152, 382 (1988).

[19] K. Kowalski and P. Piecuch, J. Chem. Phys. 115, 643 (2001).

[20] K. Kowalski and P. Piecuch, J. Chem. Phys. 113, 18 (2000).

[21] K. Kowalski and P. Piecuch, J. Chem. Phys. 120, XXXX (2004), in press.

[22] K. Kowalski and P. Piecuch, J. Chem. Phys. 115, 2966 (2001).

[23] D. R. Entem and R. Machleidt, Phys. Lett. B 524, 93 (2002).

[24] S. Weinberg, Phys. Lett. B 363, 288 (1990).

[25] U. van Kolck, Prog. Part. Nucl. Phys. 43, 337 (1999).

[26] K. Gad and H. Müther, Phys. Rev. C 66, 044301 (2002). 
[27] D. J. Dean and M. Hjorth-Jensen (2003), preprint nucl-th/0308088 